\title{
The Influence of Perceived Stress on the Alcohol Consumption of the Filipino Migrant Workers in South Korea
}

\author{
Mary Faith Lepaopao ${ }^{1}$, Cheong Hoon $\mathrm{Kim}^{2, *}$ and Nami Kim ${ }^{3}$ \\ ${ }^{1}$ Doctorate Student, Addiction Science Department \\ Sahmyook University (Seoul, Korea) \\ ${ }^{2}$ Assistant Professor, Addiction Science Department \\ Sahmyook University (Seoul, Korea) \\ ${ }^{3}$ Department Head, Addiction Science Department \\ Sahmyook University (Seoul, Korea) \\ ${ }^{*}$ Corresponding author's email: ckim [AT] syu.ac.kr
}

\begin{abstract}
This study explored how perceived stress and other common characteristics affected the alcohol consumption of the Filipino migrant workers in South Korea. 201 Filipino migrant workers $(78.1 \%$ male, 21.9\% female) completed the questionnaire containing the Perceived Stress Scale (PSS) for measuring perceived stress and Alcohol Use Disorders Identification Test-C (AUDIT-C) for measuring hazardous drinking. Pearson's correlation, regression analysis, T-test and ANOVA were used for statistical analysis. The results revealed that perceived stress was significantly correlated with alcohol consumption among undocumented migrant workers but not with their documented counterparts. Perceived stress also was found to have predicted alcohol consumption among the undocumented migrant workers. Significantly, documented migrant workers have lower levels of perceived stress and alcohol consumption compared to the undocumented migrant workers.
\end{abstract}

Keywords - migrant workers, alcohol consumption, perceived stress, AUDIT

\section{INTRODUCTION}

Irresponsible alcohol consumption is a major health dilemma and has an enormous impact on every aspect of the society. There is a flourishing evidence that alcohol use is a crucial contributing factor to global mortality [1,2,3]. It is responsible for the 3 million deaths a year, 6 deaths every minute - it causes mental and behavioral disorders and communicable and non-communicable diseases.[3]. Aside from its adverse effect on health, alcoholism takes a toll on social relationships, economy, and the society. $[4,5,6]$.

Alcohol consumption is rampant among migrant workers [7,8]. Migrant workers are more likely to experience stressful events due to many unfavorable conditions $[9,10]$. Korea is a country that has a heavy drinking culture $[11,12]$ and this culture can cross the industrial world of migrant workers. Under the report of Republic of Korea ministry of justice in 2015, there are around 570,000 low-skilled migrant workers in South Korea excluding those who are working illegally [13]. Migrant workers play an important role in keeping economic progress in Korea [14], thus, they have become the backbone of Korean industries. But it is sad that many are subjected to an abusive work condition, Little or no protective equipment when working with heavy machineries and dangerous chemicals, night shifts and long hours with a lesser wage compared to their Korean counterparts, and inadequate medical services [15]. With their status, migrant workers are at the greater risk for exploitation $[10,16)$. With the significant level of mental and emotional stress they experience daily, it is not impossible for them to turn into alcohol to cope up.

There is a growing body of literature that explores the connection between perceived stress and alcohol consumption $[17,18,19,20$,$) . For example, a study done by Heidi Hutton and company [21] in 2017$ suggests that alcohol use is positively correlated with stress among heavy drinking HIV-infected women. A different study [22] among college students' samples in the University of Connecticut suggests that alcohol is consumed more on days perceived as stressful. Another research involving 8,057 Korean subjects, ages beginning 20 years old, has shown that individuals with high perceived stress are more likely to consume alcohol [18]. Similarly, perceived level of stress was positively associated with alcohol consumption in a study involving Korean college students from 14 universities in Korea [20]. A crosssectional study among university students in France also revealed that perceived stress has made the rate of alcohol disorders among students increased [23]. 
Migrants workers are susceptible to stress brought about by a hard-living condition; distance from family members and culture adjustments [9]. Drinking is often seen as a coping mechanism and outlet for stress [24]. Latino farm workers, for example, have very high rates for alcohol dependence [25]. In China, Risky drinking practices among female migrants' women in entertainment venues are prevalent [26]. Also, a significant number of migrant workers in Spain are identified as hazardous drinkers [27]

On recognizing the increased health risk brought about by alcohol consumption among migrant workers, the interest to study the impact of perceived stress on alcohol consumption among migrant workers was heightened. Many research studies have been conducted to explore the relationship among perceived stress and alcohol consumption. However, such study among migrant workers is limited. Furthermore, very little research, if not nonexistent, has been reported among Filipino migrant workers in Korea.

Therefore, this study aimed to find out the influence of perceived stress on the alcohol consumption of the Filipino migrant workers in Korea.

\section{METHOD}

\subsection{Participants}

The subjects consisted of 201 Filipino migrant workers in South Korea. The sample included documented (21.9\%) and undocumented, (78.1) low-skilled migrant workers from different industries mostly coming from the outskirts of Seoul. $157(78.1 \%)$ were males and $44(21.9 \%)$ were females, $72(35.8 \%)$ were single, $107(53.2 \%)$ were married, $15(7.5 \%)$ were separated, and 7(3.5\%) were either annulled or divorced.

\subsection{Instruments}

Perceived stress scale (PSS) developed by Cohen [28] is among the most widely used measures of stress. This scale comes in 3 versions; 14 -item scale, 10-item scale, and the 4-item scale. All three versions were found to be valid and reliable, with the 10-item scale considered to be superior [29]. The participants were asked to evaluate their feelings and thoughts during the last month based on a five-point Likert scale (0-never, 1- almost never, 2- sometimes, 3-fairly often, $4 \mathrm{~s}-$ most often). Scores for positively worded items (4,5,7, and 8) were reverse and added to the score of the rest of the items. The total score of the 10 questions varies from $0-40,0-13=10$ perceived stress, $14-26=$ moderate perceived stress, and 27-40=high perceived stress). The Cronbach's $\alpha$ of the PSS used in Cohens' study was .78. and was initially .504 in our study. After performing a factor analysis using principal component extraction method with a varimax rotated solution and excluding the 2 items which did not attribute Cronbach's alpha, the Cronbach's $\alpha$ increased to .623. Hence, those items were not included in all the statistical analysis done in this study.

Alcohol Use disorders Identification Test-C (AUDIT-C) Is a 3-item measure from a modified version of 10-item AUDIT instrument. It is an effective tool to measure problematic drinking [30]. The total score of 3 items varies from 0 12. A score of 4 or more in men and 3 or more in women is considered hazardous drinking. In our study, we found satisfactory Cronbach's alpha values (.075) for AUDIT-C, while it was .098 in the previous study done in Japan by Osaki and company [31].

\subsection{Procedure}

This cross-sectional survey was performed among migrant workers in South Korea. The participants in this study were recruited using a non-probability sampling method in various Filipino migrant workers' communities. This method was used because probability sampling was difficult to carry out for such a big population of Filipino migrant workers. The gathering of data, which lasted for 6 months, was done by visiting Filipino communities. The Questionnaires, Alcohol Use Disorders Identification Test (AUDIT)and the Perceived stress scale were given to the respondents along with an accompanying letter containing the purpose of the study, informed consent, and confidentiality assurance. The questionnaire, anonymously taken, took approximately 5-7 minutes to complete. Important aspects of biographical information of the respondents like age, gender, age, visa status and marital status were included.

\subsection{Data Analysis}

The data was analyzed using SPSS 21.0. Descriptive statistics was run on all the variables in this study. First, differences among general characteristic were calculated using T-test and ANOVA. Correlation between variables was calculated using Pearson correlation coefficients and the predictive analysis of the independent variable was analyze using simple linear regression

\section{RESULT}

The general characteristics of the samples are described in table 1. In total, 201 subjects were involved in the study, including $157(78.1 \%)$ males and $44(21.9 \%)$ females. Approximately $90 \%$ of the respondents were in the age range $25-$ 
$34(42.8 \%, \mathrm{n}=86)$ years old and 35-44 $(44.8 \%, \mathrm{n}=90)$ years old. The other groups were in the range $18-24(5 \%, \mathrm{n}=10)$ and 44 or up $(7.5 \%, n=15)$. For marriage status, $35.8 \%(n=72)$ were single and $53.2 \%(n=107)$ were married. The rest were either estranged, annulled or divorced $(11.0 \%, \mathrm{n}=22)$. More than fifty percent $(57.2 \%, \mathrm{n}=115)$ of the respondents had stayed in Korea for not more than 5 years, 20.9\% $(n=42)$ had stayed between $6-10$ years, and $21.9 \%(n=44)$ had stayed for 11 years or more. Among 201 respondents, $157(78.11 \%)$ were documented while the rest were undocumented $(21.89 \%, \mathrm{n}=44)$.

Table 1: General Characteristics of the participants

\begin{tabular}{cccc}
\hline Variable & Group & Frequency $(\mathbf{n})$ & Percentage \% \\
\hline Gender & Male & 157 & 78.1 \\
$(\mathrm{n}=201)$ & Female & 44 & 21.9 \\
Age & & & 5.0 \\
$(\mathrm{n}=201)$ & $18-24$ & 10 & 42.8 \\
& $25-34$ & 86 & 44.8 \\
& $35-44$ & 90 & 7.5 \\
Marital Status & 44 or up & 15 & 35.8 \\
$(\mathrm{n}=201)$ & Single & 72 & 53.2 \\
& Married & 107 & 7.5 \\
Length of Stay & Estranged & 15 & 3.5 \\
$(\mathrm{n}=201)$ & Annuled/divorced & 7 & 57.2 \\
& 0-5 & 115 & 20.9 \\
Visa & 6-10 years & 42 & 21.9 \\
& 11 or more & 44 & 78.11 \\
& Documented & 157 & 21.89 \\
\hline
\end{tabular}

Figure 1 shows patterns of perceived stress and alcohol consumption. It is shown that 96.07\% (193) of the respondents were under stress-86.57\% $(\mathrm{n}=174)$ were under moderate stress and $9.50 \%(\mathrm{n}=19)$ were under high stress. For alcohol consumption, $52.24 \%(\mathrm{n}=105)$ of the respondents reported consuming alcohol at a low-risk level while $47.76 \%$ $(\mathrm{n}=96)$ reported consuming alcohol at a hazardous level.
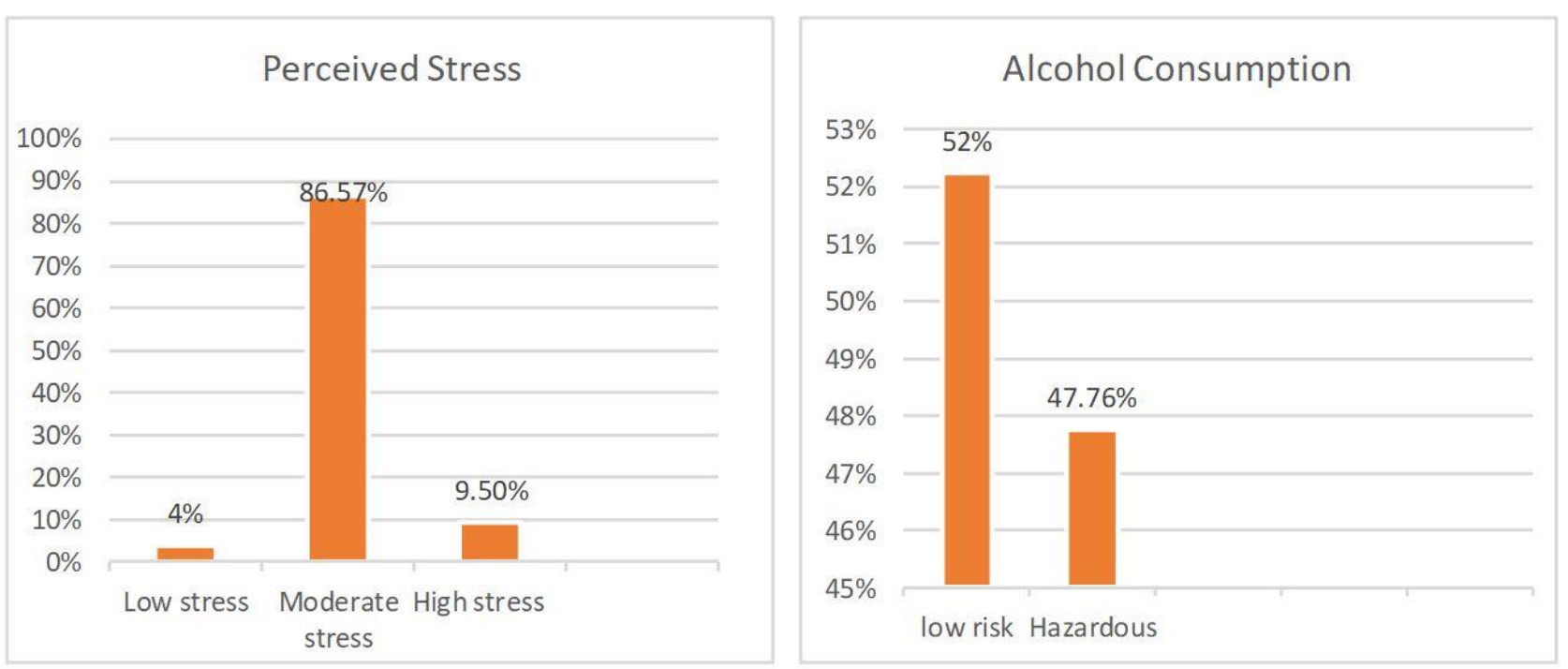

Figure 1: Patterns of perceived stress and alcohol consumption

The Comparison of the means and standard Deviations of the respondents' general characteristic is presented in Table 2. Though, there was no significant difference $(\mathrm{p}=.056)$ between male and female on alcohol consumption, it is noticeable that Filipino male migrant workers had higher alcohol consumption $(M=3.44, S D=2.70)$ than Filipino female migrant workers. $(\mathrm{M}=2.55, \mathrm{SD}=2.79)$. Though the overall perceived stress of female Filipino migrant workers was higher 
$(\mathrm{M}=14.64, \mathrm{SD}=4.36)$ than their male counterparts $(\mathrm{M}=13.84, \mathrm{SD}=3.91)$, the difference was not statistically significant $(\mathrm{p}=.246)$.

Significant difference was not found on variables perceived stress $(\mathrm{p}=.073)$ and alcohol consumption $(\mathrm{p}=.284)$ across all age groups. It is however, important to note that participants in the range 18-24 years old had noticeably lower perceived stress level $(\mathrm{M}=11.00, \mathrm{SD}=4.35)$ than the rest of the participants in the other age groups $(\mathrm{M}=14.33, \mathrm{SD}=3.60$; $\mathrm{M}=14.19, \mathrm{SD}=4.19 ; \mathrm{M}=13.20, \mathrm{SD}=4.48)$.

There was no significant difference in all marital status groups in perceived stress $(\mathrm{p}=.464)$ and alcohol consumption $(\mathrm{p}=.679)$.

Undocumented participants reported higher perceived stress $(\mathrm{M}=15.27, \mathrm{SD}=4.74)$ and higher alcohol consumption $(\mathrm{M}=4.57, \mathrm{SD}=3.08)$ compared to documented participants $(\mathrm{M}=13.66, \mathrm{SD}=3.73, \mathrm{M}=2.87, \mathrm{SD}=2.51)$. The differences between the two groups in two variables were found to be significant $(\mathrm{p}=.018, \mathrm{p}=.000)$

Length of stay in Korea did not make a significant impact on perceived stress $(\mathrm{p}=.571)$ and alcohol consumption $(\mathrm{p}=.164)$.

Table 2: Comparison of the means and standard deviations by participants' general characteristics

\begin{tabular}{|c|c|c|c|c|c|c|c|}
\hline \multicolumn{2}{|c|}{ Variable } & \multicolumn{3}{|c|}{ Stress } & \multicolumn{3}{|c|}{ Alcohol } \\
\hline & & $\mathrm{M} \pm \mathrm{SD}$ & t or $F$ & $\mathrm{p}$-value & $\mathrm{M} \pm \mathrm{SD}$ & t or $F$ & p-value \\
\hline \multirow[t]{2}{*}{ Gender } & Male (157) & $13.84 \pm 3.91$ & -1.163 & .246 & $3.44 \pm 2.70$ & 1.923 & .056 \\
\hline & Female (44) & $14.64 \pm 4.36$ & & & $2.55 \pm 2.79$ & & \\
\hline \multirow[t]{4}{*}{ Age } & $18-24(10)$ & $11.00 \pm 4.35$ & 2.362 & .073 & $2.56 \pm 2.35$ & 1.275 & .284 \\
\hline & $25-34(86)$ & $14.33 \pm 3.60$ & & & $3.57 \pm 2.90$ & & \\
\hline & $35-44(90)$ & $14.19 \pm 4.19$ & & & $3.16 \pm 2.69$ & & \\
\hline & 45 or older $4(15)$ & $13.20 \pm 4.48$ & & & $2.27 \pm 1.98$ & & \\
\hline \multirow{4}{*}{ Marital Status } & Single (72) & $14.50 \pm 3.89$ & .858 & .464 & $3.37 \pm 2.91$ & .506 & .679 \\
\hline & Married (107) & $13.64 \pm 3.79$ & & & $3.05 \pm 2.53$ & & \\
\hline & Estranged (15) & $13.87 \pm 5.76$ & & & $3.80 \pm 2.96$ & & \\
\hline & Annulled/Divorced (7) & $15.14 \pm 4.56$ & & & $3.71 \pm 3.68$ & & \\
\hline \multirow{2}{*}{ Visa status } & Undocumented (44) & $15.27 \pm 4.74$ & 2.379 & .018 & $4.57 \pm 3.08$ & 3.772 & .000 \\
\hline & Documented (157) & $13.66 \pm 3.73$ & & & $2.87 \pm 2.51$ & & \\
\hline \multirow[t]{3}{*}{ Length of Stay } & $0-5(115)$ & $13.97 \pm 3.80$ & .563 & .571 & $2.92 \pm 2.83$ & 1.823 & .164 \\
\hline & $6-10(42)$ & $13.62 \pm 5.04$ & & & $3.67 \pm 2.52$ & & \\
\hline & 11-more (44) & $14.52 \pm 3.47$ & & & $3.66 \pm 2.61$ & & \\
\hline
\end{tabular}

Correlation between perceived stress and alcohol consumption in documented and undocumented Filipino Migrant workers is presented in Table 3. There was no correlation found between perceived stress and alcohol consumption in documented Filipino migrant workers $(\mathrm{r}=.077, \mathrm{p}=>.05)$. However, a significant relation was detected in their undocumented counterparts $(\mathrm{r}=.347, \mathrm{p}=<.05)$.

Table 3: The Correlation between perceived stress and alcohol consumption in documented and undocumented Filipino Migrant workers.

\begin{tabular}{cccc}
\hline Visa Status & Variable & Perceived Stress & Alcohol consumption \\
\hline Documented & Perceived Stress & 1 & 1 \\
& Alcohol consumption & .077 & 1 \\
Undocumented & Perceived Stress & 1 & 1 \\
& Alcohol consumption & $.347 *$ &
\end{tabular}

Table 4 shows that perceived stress had a significant influence on alcohol consumption among undocumented migrant workers. $(\beta=.347, \mathrm{p}=0.025)$. The overall regression model was significant $(\mathrm{p}=.021)$. The predictor, perceived stress, account for $12 \%$ the variances in alcohol consumption. 
Table 4: Linear analysis for the variable perceived stress and alcohol consumption among undocumented Filipino migrant workers

\begin{tabular}{cccccc}
\hline \multirow{2}{*}{ Variable } & \multicolumn{2}{c}{ Unstandardized coefficients } & Standardized coefficients & \multirow{2}{*}{ t } \\
\cline { 2 - 5 } & $\mathrm{B}$ & Standard error & $\beta$ & $2.295^{*}$ \\
\hline Perceived stress & .225 & .094 & .347 & \\
\hline & $\mathrm{R}^{2}=.120$ & $\mathrm{~F}=5.738$ & $\mathrm{P}=.021^{\mathrm{b}}$ & &
\end{tabular}

\section{DISCUSSION}

As far as our knowledge is concerned, there is very little study, if not non-existent, done among Filipino migrant workers in Korea, with its focus on the impact of perceived stress on alcohol consumption. It has been known that alcohol consumption is a global health problem $[1,2]$ and it is prevalent among low-skilled migrant workers [6,7]. One of the factors that has been established to affect alcohol consumption is perceived stress. Low-skilled migrant workers may experience high levels of stress related to the difficult nature of their job. $[9,14,15]$

This study aimed at exploring the prevalence of perceived stress and alcohol consumption of Filipino low-skilled migrant workers in Korea., at the same time also finding out the differences of perceived stress and alcohol consumption in relation to personal characteristics. It also aimed to find out the relationship of perceived stress and alcohol consumption, whilst also finding out whether perceived stress is a predictor of alcohol consumption.

It is shown that $96.07 \%$ (193) of the respondents were under stress-86.57\% ( $\mathrm{n}=174)$ were under moderate stress and $9.50 \%(\mathrm{n}=19)$ were under high stress. The number of migrant workers who were under high stress in our study was significantly lower than migrant workers in Malaysia in the study led by Foo [32]. They reported more than 85\% of their respondents had higher than average perceived stress. The difference could be attributed to various social aspects of migrant workers living in South Korea, which differs from those migrant workers living in Malaysia.

Almost half of the participants in this study $(47.76, \mathrm{n}=96)$ were consuming alcohol at hazardous level. This result is a little higher compared to a related study on alcohol consumption among migrant workers in Shanghai which reported $37.7 \%$ were consuming alcohol at hazardous level [33]. Korea's drinking culture could be the contributing factor to the higher alcohol consumption among migrant workers in Korea compared to the migrant workers in Shanghai.

Though it is notable that alcohol consumption in our study were much higher among male than their female counterparts, the difference was not significant $(\mathrm{p}=.056)$. This could be explained by the fact that women have begun to mirror drinking patterns once known to men. The finding in the study led by Tim Lade shows that the gap between men and women's drinking habits is narrowing [34].

Our results also revealed a significant difference in visa status on perceived stress and alcohol consumption. Undocumented workers showed a significantly higher perceived stress and higher alcohol consumption than documented workers. Unfavorable circumstances surrounding illegal migrant workers such as lack of legal protection, lower compensation and fewer rights may explain this finding [15]. In Addition, Atteyara [35] mention in his study that documented migrant workers in Korea do not have medical insurance, live in fear of being caught by police, succumb to late or unpaid wages, and deal with abusive employers.

Length of stay was not a significant factor in perceived stress and alcohol consumption. A closely related study by Doki and her companions [36] found similar result to ours. They reported that in their study length of stay was not a significant factor for stress.

It was somewhat expected to find a significant relationship on perceived stress and alcohol consumption. The result of our study supported the result of the previous study by Yoon and company [17] involving more than 8000 thousand Korean subjects, ages 20 and above. They reported that individuals with high perceived stress are more likely to consume alcohol. Another study involving Korean students also revealed that alcohol misuse was associated with perceived stress. [23] More initial findings also found similar results to ours [10,17,23,37,38,39]. However, in our study, such significant relationship, was only found in undocumented migrant workers and not in documented migrant workers. It is also important to note that in our study, participants drink even when they are just moderately stressed. Moreover, the result of this study shows that an increase in perceived stress led to an increase in alcohol consumption.

The influence of perceived stress on alcohol consumption among the undocumented workers is significant. Results of the regression analysis revealed that perceived stress is a predictor of alcohol consumption. Though it only accounts for $12 \%$ the variance in alcohol consumption, it remains a meaningful result since human behavior is harder to predict than physical processes. Therefore, we believe this result is significantly meaningful. 


\section{LIMITATION OF THE STUDY}

Our study has limitations. First, the sampling method was convenience sampling, so it may not be fully representing the larger population of Filipino migrant workers in Korea. Furthermore, it does not include other nationalities, and therefore should not be considered a representation of the general population of migrant workers in Korea.

Another limitation was the deletion of the 2 items from the 10-item Perceived Stress Scale. The initial reliability test resulted to a less than .05 Cronbach alpha, so the researchers opted to delete the said items to improve overall reliability.

Despite these limitations, the study findings give a contribution to literature in the areas of mental health and health behaviors among migrant workers, and thus may be useful for professionals in helping migrant workers who are struggling with stress and drinking problems.

\section{CONCLUSION AND RECOMMENDATION}

In conclusion, it has been found that most Filipino migrant workers are moderately stressed. However, quite a number of participants are engaged in hazardous drinking. This study would like to emphasize that alcohol consumption can increase with the increase in perceived stress, regardless of the person's perceived stress level. Many participants were moderately stressed, yet they engaged in hazardous drinking. It can be inferred that drinking can be their way of coping up with the challenges, regardless of how they perceived stress. Hence, it is necessary for them to develop other effective coping skills so they will not turn to harmful sources of stress relief.

A very significant finding showed that undocumented Filipino migrant workers were found to be significantly more stressed and more prone to drinking alcohol compared to documented Filipino migrant workers. The findings in this study may be very relevant in providing a basis in developmental programs for migrant workers wellbeing, especially the marginalized undocumented workers. It is recommended that migrant centers in Korea create programs and provide services specifically catering undocumented migrant worker. With this effort, inequity gap between documented and undocumented migrant workers may be lessened.

The relationship of perceived stress and alcohol consumption, though weak, was significant. It has been found also that perceived stress is a significant predictor of alcohol consumption. Therefore, it is not something to be taken for granted. Still, preventive and intervention programs like providing classes on stress management and stress reduction should be made available to reduce the future burden of alcohol related to stressful events.

To provide a strong foundation for public health policies and intervention on alcohol consumption among migrant workers, future research studies should continue to further investigate major risk factors and protective factors that may contribute to or prevent hazardous alcohol consumption.

\section{ACKNOWLEDGEMENT}

We express our gratitude all migrant workers from different parts of South Korea who participated in our study. We also give thanks to the Addiction Science Department of Samhyook University for the Guidance. Authors have no conflicts of interest to report.

\section{REFERENCES}

[1] Moss, H., "The Impact of Alcohol on Society: A Brief Overview”, Social Work in Public Health, vol. 28. pp175-177, 2013.

[2] Rehm, J., Shield, K., "Global alcohol-attributable deaths from cancer, liver cirrhosis, and injury in 2010", Alcohol Research: Current Reviews, vol. 35. no. 2, pp.174-183, 2013.

[3] World Health Organization. Global status report on alcohol and health 2018: Vladimir Poznyak and Dag Rekver. Geneva, Switzerland: World health Organization, 2018. Available from: https://www.who.int/substance_abuse/publications/global_alcohol_report [Accessed 21 May 2019]

[4] Rehm, J., Baliunas, D., Borges, G. L., Graham, K., Irving, H., Kehoe, T., Taylor, B., "The relation between different dimensions of alcohol consumption and burden of disease: An overview", Addiction, vol. 105, no.5, pp.817-843, 2010.

[5] Casswell, S., Tamarangsi, T., "Reducing harm from alcohol: call to action”, Alcohol and Global Health, vol. 373 , no. 9682, pp. 2247-2257, 2009.

[6] Cameron, D., "Mapping Social Consequences of Alcohol Consumption", Alcohol and Alcoholism, volume 37, no. 1, pp. 103-104, 2002. 
[7] Duke, M. R., Gómez Carpinteiro, F. J. "The Effects of Problem Drinking and Sexual Risk Among Mexican Migrant Workers on Their Community of Origin”, Human Organization, vol. 68, no. 3, p. 328,2009.

[8].Soe, T.J., Hongsranagon, P., Havanond,P, "Determinants and consequences of alcohol consumption among male adult Myanmar migrant workers in Ratchaburi province”, J Health Res, vol. 26, pp.27-32, 2012.

[9] Ward, C., Bochner, S., Furnham, A., The Psychology of Culture Shock, Routledge, Sussex, 2015

[10] Zhong, B. L., Liu, T. B., Huang, J. X., Fung, H. H., Chan, S. S., Conwell, Y., Chiu, H. F., “Acculturative stress of Chinese rural-to-urban migrant workers", A Qualitative Study, vol.11, no.6, e0157530. 2016

[11] Reports about alcoholic violence reach 360 thousand cases at every year: $26.6 \%$ of police work. Chosunilbo; [accessed on 24 May 2019]. Available at http://news.chosun.com/site/data/html_dir/2012/05/31/2012053100164.html.

[12] Koreans' Alcohol Consumption Doubles in Past 50 Years. Chusunilbo; [accessed on 24 May 2019]. Available at http://english.chosun.com/site/data/html_dir/2017/06/26/2017062601308.html

[13] Republic of Korea Ministry of Justice, Immigration Service, Employment Permit System (EPS) website, [accessed May 24, 2019] . Available at https://www.eps.go.kr/eo/main.eo

[14] Yi, K., Cho, H., "The survey study on working conditions and industrial safety \& health of foreign workers", Journal of the Korea Safety Management and Science, vol. 14, no.1, 2012

[15] Gushulak, B.D., Macpherson, D.W., "The basic principles of migration health: population mobility and gaps in disease prevalence", Emerg Themes Epidemiol, vol, 3, no.3, 2006

[16] Mohammad, A.A., "Ending Exploitation of Migrant Workers", The Fletcher Forum of World Affairs, vol., 34, no.2, pp. 87-108, 2010

[17] Yoon, S.J., Kim, H.J.,Doo M., “Association between perceived stress, alcohol consumption levels and obesity in Koreans", Asia Pac J Clin Nutr, vol. 25, no.2, pp. 316-325

[18] Sacco, P., Bucholz, K.K, Harrington, D, "Gender differences in stressful life events, social support, perceived stress, and alcohol use among older adults: results from a National Survey”, Subst Use Misuse, vol.49, no.4, pp. 456-465.

[19] Jang, S. M., Sohn, S., Yu, M., "Perceived stress, alcohol consumption, and internet use among Korean college students: Does sensation-seeking matter?", Children and Youth Services Review, pp.354-360, 2018

[20] Sebena, R., El Ansari, W., Stock, C., Orosova, O., Mikolajczyk, R.T., “Are perceived stress, depressive symptoms and religiosity associated with alcohol consumption? A survey of freshmen university students across five European countrie", Subst Abuse Treat Prev Policy, vol.7, no21, 2012

[21] Hutton, H.E., Lesko, C., Chander, G., Lau, B.M., Wand, G.S., McCaul, M.E., "Differential effects of perceived stress on alcohol consumption in moderate versus heavy drinking HIV-infected women", Drug and Alcohol Dependence, vol. 178, pp. 380-385, 2013

[24] Aldridge-Gerry, A.A., Roesch, S.C., Villodas, F., McCabe, C., Leung, Q.K., Da Costa, M. "Daily stress and alcohol consumption: modeling between-person and within-person ethnic variation in coping behavior", J Stud Alcohol Drugs, vol.72, no.1, pp.125-134, 2011

[25] Sanchez, J., “Alcohol use among Latino migrant workers in South Florida”, Drug and Alcohol Dependence, vol. 151, pp. 241-249, 2015.

[26] Zaller, N., Huang, W., He, H., Dong, Y., Song, D., Zhang, H., Operario, D., "Risky alcohol use among migrant women in entertainment venues in China", Alcohol and Alcoholism, vol. 49, no.3, pp. 321-326, 2014

[27] Perez-Carceles, M. D., Medina, M. D., Perez-Flores, D., Noguera, J. A., Pereniguez, J. E., Madrigal, M., Luna, A., "Screening for hazardous drinking in migrant workers in Southeastern Spain", Journal of Occupational Health, vol. 56, no.1, pp. 39-48, 2014

[29] Cohen, J. (1988). Statistical Power Analysis for the Behavioral Sciences, Lawrence Erlbaum Associates, Publishers., Hillsdale, NJ: New Jersey, 1988

[30] Bush K., Kivlahan D.R., McDonell M..B, Fihn S.D., Bradley K.A, “The AUDIT alcohol consumption questions (AUDIT-C): an effective brief screening test for problem drinking. Ambulatory care quality improvement project (ACQUIP). Alcohol use disorders identification test”, Arch Intern Med., vol.158, no. 16, pp. 1789-1795

[31] Osaki, Y., Ino, A., Matsushita, S., Higuchi, S., Kondo, Y., Kinjo, A., "Reliability and validity of the alcohol use disorders identification test - consumption in screening for adults with alcohol use disorders and risky drinking in Japan. Asian Pacific Journal of Cancer Prevention, vol. 15, no.16, pp. 6571-6574, 2014

[32] Foo, A., Jeevanaraj, P., Beatrice, A., Pereira, A., \& Fatima, M.A., "Stress levels and its associated factors among Nepali, Bangladeshi and Indonesian migrant workers in the Klang Valley, Selangor, Malaysia. Journal of Management and Science. Vol. 15, pp.120-134, 2017

[33] Yang, H., Gao, J., Wang, T., Yang, L., Liu, Y., Shen, Y., Zhu, S., “Association between adverse mental health and an unhealthy lifestyle in rural-to-urban migrant workers in Shanghai", Journal of the Formosan Medical Association, vol.116, no.2, pp. 90-98. 2017

[34] Lade, T., Chapman, C., Swift, W., Keyes, K., Tonks, Z., Teesson, M., "Birth cohort trends in the global epidemiology of alcohol use and alcohol-related harms in men and women: Systematic review and metaregression. BMJ Open,vol.6, no.10, 2016 
[35]Atteraya, M., \& Ji Myoung, J., Eun Lee, D., Hang Ryun, J., \& Gnawali, S., “Social Adjustment for undocumented migrant workers in South Korea: Role of religious institutions", Journal of International studies, vol. 23., pp. 53-80, 2015.

[36] Doki, S., Sasahara, S., Matsuzaki, I., "Stress of working abroad: a systematic review”, Int Arch Occup Environ Health, vol. 91, no.7, pp. 767-784, 2018

[37] Bai, A., Li, H., Huang, Y., Liu, X., Gao, Y., Wang, P., Chen, K. “A survey of overall life satisfaction and its association with breast diseases in Chinese women", Cancer Medicine, vol.5, no.1, pp. 111-119, 2017

[39] Newcomb, M. D., Bentler, P.M., Collins C., "Alcohol use and dissatisfaction with self and life: A longitudinal analysis of young adults”, Journal of Drug Issues, vol. 16, no.4, pp.479-494, 1986 